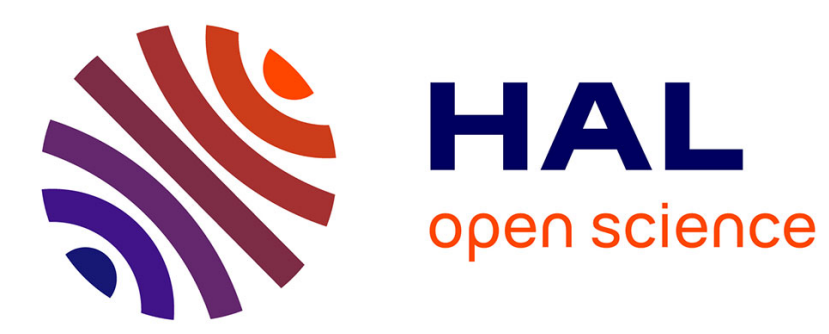

\title{
FRACTURE AND PLASTIC MODELS AS $\Gamma$-LIMITS OF DAMAGE MODELS UNDER DIFFERENT REGIMES
}

Flaviana Iurlano

\section{- To cite this version:}

Flaviana Iurlano. FRACTURE AND PLASTIC MODELS AS $\Gamma$-LIMITS OF DAMAGE MODELS UNDER DIFFERENT REGIMES. Advances in Calculus of Variation, 2013, 6 (2), 10.1515/acv-20110011. hal-02145309

\author{
HAL Id: hal-02145309 \\ https://hal.science/hal-02145309
}

Submitted on 2 Jun 2019

HAL is a multi-disciplinary open access archive for the deposit and dissemination of scientific research documents, whether they are published or not. The documents may come from teaching and research institutions in France or abroad, or from public or private research centers.
L'archive ouverte pluridisciplinaire HAL, est destinée au dépôt et à la diffusion de documents scientifiques de niveau recherche, publiés ou non, émanant des établissements d'enseignement et de recherche français ou étrangers, des laboratoires publics ou privés. 


\title{
FRACTURE AND PLASTIC MODELS AS T-LIMITS OF DAMAGE MODELS UNDER DIFFERENT REGIMES
}

\author{
Flaviana IURLANO \\ SISSA, Via Bonomea 265 \\ 34136 Trieste, Italy
}

\begin{abstract}
We consider a variational model for damaged elastic materials. This model depends on three small parameters, which are related to the cost of the damage, to the width of the damaged regions, and to the minimum elasticity constant attained in the damaged regions. As these parameters tend to zero, our models $\Gamma$-converge to a model for brittle fracture, for fracture with a cohesive zone, or for perfect plasticity, depending on the asymptotic ratios of the three parameters.
\end{abstract}

\section{INTRODUCTION}

In this paper we analyze the asymptotic behavior of particular damage models for linearly elastic materials. The standard presentations of damage problems describe the state of the elastic body by means of two functions: the displacement $u$ and an internal variable $v$, which is defined on the reference configuration $\Omega \subset \mathbb{R}^{n}$ with values in the interval $[0,1]$. We assume that $v=1$ corresponds to the original elastic material, while $v=0$ represents the totally damaged material, which is unable to resist any force. The elasticity tensor depends continuously and monotonically on the variable $v$.

We consider a homogeneous isotropic material, which remains isotropic during the process, and we face the antiplane case; by these assumptions the displacement $u$ is scalar and the elasticity tensor reduces to a single constant, which will be chosen as the internal variable $v$ of the problem. Then, the stored elastic energy related to the displacement $u$ and to the internal variable $v$ is

$$
\int_{\Omega} v|\nabla u|^{2} d x
$$

In the simplest model the energy dissipation due to the damage is given by

$$
\int_{\Omega} a(1-v) d x
$$

for a suitable constant $0<a<\infty$.

Reasonably, our model guarantees some regularity in the distribution of the damage. This is obtained through the penalization term on the spatial variations of $v$

$$
\int_{\Omega} b|\nabla v|^{p} d x
$$

for a suitable constant $0<b<\infty$ and for $p>1$. We note that in [10] a different bound on $\nabla v$ was introduced, i.e. the constraint

$$
|\nabla v| \leq c \quad \mathcal{L}^{n} \text {-a.e. in } \Omega
$$

for some $c<\infty$. In fact the previous constraint can be formally seen as the limiting case of (1.2) when $p \rightarrow \infty$.

We also assume that the material is never totally damaged and we require

$$
d \leq v \leq 1
$$

where $d$ is a positive constant.

2010 Mathematics Subject Classification. Primary: 74A45, 74C99, 49J45; Secondary: 35A35.

Key words and phrases. Damage problems, fracture mechanics, cohesive zone models, Hencky's plasticity model, $\Gamma$-convergence. 
Therefore, the total energy is given by

$$
\int_{\Omega} v|\nabla u|^{2} d x+\int_{\Omega} a(1-v) d x+\int_{\Omega} b|\nabla v|^{p} d x
$$

where $v$ satisfies (1.3). This functional is complemented by suitable boundary conditions and lower order terms due to the action of external forces. The damage model related to (1.4) in fact belongs to the class of models studied in [12].

The purpose of this paper is to study the limit behavior of the energies (1.4) as $a \rightarrow \infty, b \rightarrow 0$, and $d \rightarrow 0$. Since it does not introduce additional difficulties in our analysis, we replace the function $1-v$ in (1.4) by an arbitrary strictly decreasing continuous function $\psi$ on $[0,1]$ with $\psi(1)=0$. In order to state precisely our results we introduce three sequences $\delta_{k}, \epsilon_{k}, \eta_{k}>0$ with $\delta_{k} \rightarrow 0, \epsilon_{k} \rightarrow 0$, $\eta_{k} \rightarrow 0$. It is not restrictive to assume that $\eta_{k} / \delta_{k} \rightarrow \alpha$ and $\delta_{k} / \epsilon_{k} \rightarrow \beta$, with $0 \leq \alpha, \beta \leq \infty$. For $u \in H^{1}(\Omega)$ and $v \in W^{1, p}(\Omega)$ with

$$
\eta_{k} \leq v \leq 1
$$

we define

$$
F_{k}(u, v):=\int_{\Omega}\left(v|\nabla u|^{2}+\frac{\psi(v)}{\delta_{k}}+c \epsilon_{k}^{p-1}|\nabla v|^{p}\right) d x
$$

where $0<c<\infty$; we set $F_{k}(u, v):=+\infty$ otherwise in $L^{1}(\Omega) \times L^{1}(\Omega)$.

We determine the $\Gamma$-limit in $L^{1}(\Omega) \times L^{1}(\Omega)$ of the sequence $\left(F_{k}\right)$. We find that this limit depends on $\alpha$ and $\beta$. For some values of the parameters the limit functional is related to a fracture problem; this is due to damage concentration along the limit cracks. For some other values the limit is related to perfect plasticity; in this case we see damage diffusion, which leads to plastic strains. To describe the limit problem we need some terminology from the theory of $B V$ functions, for which we refer to [1] and to Section 2 below.

For every $0 \leq \alpha, \beta \leq \infty$ we introduce the functional $\Phi_{\alpha, \beta}: L^{1}(\Omega) \mapsto[0, \infty]$ defined as follows. In the case $0<\alpha<\infty, 0<\beta<\infty$ we define

$$
\Phi_{\alpha, \beta}(u):=\int_{\Omega}|\nabla u|^{2} d x+a_{\beta} \mathcal{H}^{n-1}\left(J_{u}\right)+b_{\alpha} \int_{J_{u}}|[u]| d \mathcal{H}^{n-1} \quad \text { for } u \in S B V^{2}(\Omega)
$$

and $\Phi_{\alpha, \beta}(u):=\infty$ if $u \notin S B V^{2}(\Omega)$. Here $\nabla u$ is the density of the absolutely continuous part of the distributional derivative of $u, \mathcal{H}^{n-1}$ is the $(n-1)$-dimensional Hausdorff measure in $\mathbb{R}^{n}, J_{u}$ is the jump set of $u,[u]$ is the jump of $u, a_{\beta}$ is a constant depending on $\beta$ and $p$, while $b_{\alpha}$ is a constant depending on $\alpha$. The precise definitions of $a_{\beta}$ and $b_{\alpha}$ are given in (3.6), (see page 6 for the case $p=\infty)$.

If $\alpha=0$ and $0<\beta<\infty$ we define

$$
\Phi_{0, \beta}(u):=\int_{\Omega}|\nabla u|^{2} d x+a_{\beta} \mathcal{H}^{n-1}\left(J_{u}\right) \quad \text { for } u \in G S B V^{2}(\Omega) \cap L^{1}(\Omega)
$$

and $\Phi_{0, \beta}(u):=\infty$ otherwise.

For $\alpha=\infty$ or $\beta=0$ we define

$$
\Phi_{\alpha, \beta}(u):=\int_{\Omega}|\nabla u|^{2} d x \quad \text { for } u \in H^{1}(\Omega)
$$

and $\Phi_{\alpha, \beta}(u):=\infty$ if $u \notin H^{1}(\Omega)$.

When $\alpha=0$ and $\beta=\infty$ we set

$$
\Phi_{0, \infty}(u):=0 \quad \text { for } u \in L^{1}(\Omega) .
$$

Finally in the case $0<\alpha<\infty$ and $\beta=\infty$ we set

$$
\Phi_{\alpha, \infty}(u):=\int_{\Omega} f_{\alpha}(|\nabla u|) d x+b_{\alpha}\left|D^{s} u\right|(\Omega) \quad \text { for } u \in B V(\Omega)
$$

and $\Phi_{\alpha, \infty}(u):=\infty$ if $u \notin B V(\Omega)$. Here $f_{\alpha}(t):=t^{2}$ for $0 \leq t<b_{\alpha} / 2, f_{\alpha}(t):=b_{\alpha}\left(t-b_{\alpha} / 4\right)$ for $t \geq b_{\alpha} / 2$, and $D^{s} u$ is the singular part of the distributional derivative of $u$.

We prove the following theorem (see Theorem 3.1). 
Theorem 1.1. The $\Gamma$-limit of $\left(F_{k}\right)$ in $L^{1}(\Omega) \times L^{1}(\Omega)$ is the functional $F_{\alpha, \beta}$ defined by

$$
F_{\alpha, \beta}(u, v):= \begin{cases}\Phi_{\alpha, \beta}(u) & \text { if } v=1 \mathcal{L}^{n} \text {-a.e. in } \Omega, \\ +\infty & \text { otherwise. }\end{cases}
$$

The functional $F_{\alpha, \beta}$ with $\alpha=0$ and $0<\beta<\infty$ is related to variational models for brittle fracture (see [5]), whereas for $0<\alpha<\infty$ and $0<\beta<\infty$ it is related to models for fracture with a cohesive zone and also to models for plastic slips (see [2]). When $\alpha=\infty$ or $\beta=0$ the limit functional corresponds to an elasticity problem without cracks. Finally when $0<\alpha<\infty$ and $\beta=\infty$ the functional $F_{\alpha, \infty}$ is related to the Hencky's plasticity model (see, for instance, [11]), so that we are able to simulate a plastic material with damaged elastic materials.

Our functionals in the regime corresponding to $\alpha=0$ and $0<\beta<\infty$ are essentially those introduced by Ambrosio and Tortorelli in [4] (see also [3]) to approximate the Mumford-Shah functional for image segmentation. Here we extend the study of the limit behavior of these functionals to the other regimes $\alpha>0$, or $\beta=0$, or $\beta=\infty$; we provide an approximation of the functional (1.6), different from those studied in [2] and [10].

In the case $\alpha=0$ and $0<\beta<\infty$, which leads to the Mumford-Shah functional, proofs are similar to those of [4], so that they are omitted. In the case $0<\alpha<\infty, 0<\beta<\infty$, which gives a fracture model with a cohesive zone, some arguments of [4] and of [10] still work; for clearness we sketch them, but we provide details only for original arguments. The case $0<\alpha<\infty, \beta=\infty$, which leads to perfect plasticity, requires new strategies.

Through Theorem 3.1 we prove the following result about the limit behaviour of minima and minimizers of some functionals related to $F_{\alpha, \beta}$ (see Theorem 1.2).

Theorem 1.2. Let $r>1$; let $\left(\delta_{k}\right),\left(\epsilon_{k}\right)$, and $\left(\eta_{k}\right)$ be infinitesimal sequences of positive numbers, and let $g \in L^{r}(\Omega)$. For every $k$, let $\left(u_{k}, v_{k}\right)$ be a minimizer of the functional

$$
\int_{\Omega}\left(v|\nabla u|^{2}+\frac{\psi(v)}{\delta_{k}}+c \epsilon_{k}^{p-1}|\nabla v|^{p}+|u-g|^{r}\right) d x
$$

with the costraint (1.5). Then $v_{k} \rightarrow 1$ strongly in $L^{1}(\Omega)$ and a subsequence of $\left(u_{k}\right)$ converges strongly in $L^{r}(\Omega)$ to a minimizer $u$ of the following limit problem:

$$
\begin{gathered}
\min _{u \in S B V^{2}(\Omega)}\left(\int_{\Omega}|\nabla u|^{2} d x+a_{\beta} \mathcal{H}^{n-1}\left(J_{u}\right)+b_{\alpha} \int_{J_{u}}|[u]| d \mathcal{H}^{n-1}+\int_{\Omega}|u-g|^{r} d x\right), \text { if } 0<\alpha, \beta<\infty, \\
\min _{u \in G S B V^{2}(\Omega)}\left(\int_{\Omega}|\nabla u|^{2} d x+a_{\beta} \mathcal{H}^{n-1}\left(J_{u}\right)+\int_{\Omega}|u-g|^{r} d x\right), \text { if } \alpha=0,0<\beta<\infty, \\
\min _{u \in H^{1}(\Omega)}\left(\int_{\Omega}|\nabla u|^{2} d x+\int_{\Omega}|u-g|^{r} d x\right), \text { if } \alpha=\infty \text { or } \beta=0, \\
\min _{u \in L^{1}(\Omega)}\left(\int_{\Omega}|u-g|^{r} d x\right), \text { if } \alpha=0, \beta=\infty, \\
\min _{u \in B V(\Omega)}\left(\int_{\Omega} f_{\alpha}(|\nabla u|) d x+b_{\alpha}\left|D^{s} u\right|(\Omega)+\int_{\Omega}|u-g|^{r} d x\right), \text { if } 0<\alpha<\infty, \beta=\infty .
\end{gathered}
$$

Moreover for every $\alpha$ and $\beta$ the minimum values of (1.7) tend to the minimum value of the limit problem.

The paper is composed of six sections. First we introduce the problem and the notation used in the sequel (Sections 1 and 2). In Section 3 we state the $\Gamma$-convergence result, whose proof in dimension one is given in Section 4; the $n$-dimensional case is faced in Section 5 . We conclude the paper proving the convergence of minima and minimizers (Section 6).

\section{Notation And Preliminaries}

Let $n \geq 1$ be a fixed integer. The Euclidean distance of the point $x$ from the set $E \subset \mathbb{R}^{n}$ is denoted by $d(x, E)$. The Lebesgue measure and the $k$-dimensional Hausdorff measure in $\mathbb{R}^{n}$ are denoted by $\mathcal{L}^{n}$ and $\mathcal{H}^{k}$, respectively. 
Let $\Omega$ be an open subset of $\mathbb{R}^{n}$ and let $u \in B V(\Omega)$. For the definitions of the distributional gradient $D u$, the one-sided approximate limits $u^{+}$and $u^{-}$, the jump function $[u]$, the jump set $J_{u}$, and the approximate differential $\nabla u$ we refer to [1, Sections 3.1, 3.6].

By strong convergence in $B V(\Omega)$ we mean the convergence with respect to the norm

$$
\|u\|_{B V(\Omega)}:=\|u\|_{L^{1}(\Omega)}+|D u|(\Omega),
$$

whereas by weakly* convergence of $u_{k}$ to $u$ in $B V(\Omega)$ we mean the strong convergence $u_{k} \rightarrow u$ in $L^{1}(\Omega)$ joined with the weakly* convergence of $D u_{k}$ to $D u$ in $\Omega$, i.e.,

$$
\lim _{k \rightarrow \infty} \int_{\Omega} \varphi d D u_{k}=\int_{\Omega} \varphi d D u,
$$

for every continuous function $\varphi$ vanishing on $\partial \Omega$.

If $u \in B V(\Omega)$ then

$$
D u=D^{a} u+D^{s} u=D^{a} u+D^{j} u+D^{c} u,
$$

where $D^{a} u$ is absolutely continuous and $D^{s} u=D^{j} u+D^{c} u$ is singular with respect to the Lebesgue measure; in particular $D^{j} u$ denotes the jump derivative of $u$, whereas $D^{c} u$ is the Cantor part of the derivative of $u$ (see [1, Section 3.9]). In particular the approximate differential $\nabla u$ coincides $\mathcal{L}^{n}$-a.e. with the density of $D^{a} u$.

The spaces $S B V(\Omega), G B V(\Omega), G S B V(\Omega)$ are defined as in [1]. We recall that a $G B V$-function is weakly approximately differentiable $\mathcal{L}^{n}$-a.e. in $\Omega$ (see [1, Definition 4.31, Theorem 4.34]). Since an approximately differentiable function $u$ is also weakly approximately differentiable and the approximate differential coincides with the weak approximate differential $\mathcal{L}^{n}$-a.e. in $\Omega$, we also denote the weak approximate differential by $\nabla u$.

Let $p \in] 1, \infty[$; in what follows we adopt the following definitions

$$
\begin{aligned}
S B V^{p}(\Omega) & :=\left\{u \in S B V(\Omega): \nabla u \in L^{p}\left(\Omega, \mathbb{R}^{n}\right) \text { and } \mathcal{H}^{n-1}\left(J_{u}\right)<\infty\right\}, \\
G S B V^{p}(\Omega) & :=\left\{u \in G S B V(\Omega): \nabla u \in L^{p}\left(\Omega, \mathbb{R}^{n}\right) \text { and } \mathcal{H}^{n-1}\left(J_{u}\right)<\infty\right\} .
\end{aligned}
$$

We also recall that, in the case $\Omega \subset \mathbb{R}$, if $u \in S B V^{2}(\Omega)$ then $u \in H^{1}\left(\Omega \backslash J_{u}\right)$. Conversely, if $\Omega \subset \mathbb{R}$ and there exists a finite set $F$ such that $u \in H^{1}(\Omega \backslash F)$ then $u \in S B V^{2}(\Omega)$ and $J_{u} \subset F$.

For the $\Gamma$-convergence theory we refer to [9].

\section{The $\Gamma$-Convergence Result}

3.1. The case $\boldsymbol{p}<\infty$. Let $\Omega$ be a bounded open subset of $\mathbb{R}^{n}$, let $1<p<\infty$, and let $\delta_{k}>0$, $\epsilon_{k} \geq 0, \eta_{k} \geq 0$ be infinitesimal sequences. Our purpose is to study the $\Gamma$-limit in $L^{1}(\Omega) \times L^{1}(\Omega)$ of the sequence of functionals $F_{k}: L^{1}(\Omega) \times L^{1}(\Omega) \rightarrow[0, \infty]$ defined by

$$
F_{k}(u, v):= \begin{cases}\int_{\Omega}\left(v|\nabla u|^{2}+\frac{\psi(v)}{\delta_{k}}+c \epsilon_{k}^{p-1}|\nabla v|^{p}\right) d x & \text { if }(u, v) \in H^{1}(\Omega) \times V_{\eta_{k}} \\ & \text { otherwise }\end{cases}
$$

where $c>0$,

$$
\begin{aligned}
& \psi \in C([0,1]) \text { is strictly decreasing with } \psi(1)=0, \\
& V_{\eta_{k}}:=\left\{v \in W^{1, p}(\Omega): \eta_{k} \leq v \leq 1 \mathcal{L}^{n} \text {-a.e. in } \Omega\right\} .
\end{aligned}
$$

We assume that the limits

$$
\alpha:=\lim _{k \rightarrow \infty} \frac{\eta_{k}}{\delta_{k}} \quad \text { and } \quad \beta:=\lim _{k \rightarrow \infty} \frac{\delta_{k}}{\epsilon_{k}}
$$

exist. When $0<\alpha<\infty$ and $0<\beta<\infty$ we define $\Phi_{\alpha, \beta}: L^{1}(\Omega) \mapsto[0, \infty]$ by

$$
\Phi_{\alpha, \beta}(u):= \begin{cases}\int_{\Omega}|\nabla u|^{2} d x+a_{\beta} \mathcal{H}^{n-1}\left(J_{u}\right)+b_{\alpha} \int_{J_{u}}|[u]| d \mathcal{H}^{n-1} & \text { if } u \in S B V^{2}(\Omega) \\ +\infty & \text { otherwise }\end{cases}
$$


where

$$
a_{\beta}:=2\left(\frac{q}{\beta}\right)^{\frac{1}{q}}(c p)^{\frac{1}{p}} \int_{0}^{1} \psi^{\frac{1}{q}} d s, \quad b_{\alpha}:=2(\alpha \psi(0))^{\frac{1}{2}}, \quad \text { and } \quad \frac{1}{p}+\frac{1}{q}=1 .
$$

In the limiting case when $\alpha=0$ and $0<\beta<\infty$ we define

$$
\Phi_{0, \beta}(u):= \begin{cases}\int_{\Omega}|\nabla u|^{2} d x+a_{\beta} \mathcal{H}^{n-1}\left(J_{u}\right) & \text { if } u \in G S B V^{2}(\Omega) \cap L^{1}(\Omega), \\ \infty & \text { otherwise. }\end{cases}
$$

If $\alpha=\infty$ or $\beta=0$ we define

$$
\Phi_{\alpha, \beta}(u):= \begin{cases}\int_{\Omega}|\nabla u|^{2} d x & \text { if } u \in H^{1}(\Omega), \\ +\infty & \text { otherwise. }\end{cases}
$$

It remains to define the functional $\Phi_{\alpha, \beta}$ when $0 \leq \alpha<\infty$ and $\beta=\infty$. When $\alpha=0$ and $\beta=\infty$ we set

$$
\Phi_{0, \infty}(u):= \begin{cases}0 & \text { if } u \in L^{1}(\Omega) \\ +\infty & \text { otherwise }\end{cases}
$$

whereas for $0<\alpha<\infty$ and $\beta=\infty$ we set

$$
\Phi_{\alpha, \infty}(u):= \begin{cases}\int_{\Omega} f_{\alpha}(|\nabla u|) d x+b_{\alpha}\left|D^{s} u\right|(\Omega) & \text { if } u \in B V(\Omega), \\ +\infty & \text { otherwise, }\end{cases}
$$

where $f_{\alpha}(t)=t^{2}$ for $0 \leq t<b_{\alpha} / 2$ and $f_{\alpha}(t)=b_{\alpha}\left(t-b_{\alpha} / 4\right)$ for $t \geq b_{\alpha} / 2$.

The following $\Gamma$-convergence result holds.

Theorem 3.1. Assume (3.1)-(3.4) and assume that $\Omega$ has Lipschitz boundary. The $\Gamma$-limit of $\left(F_{k}\right)$ in $L^{1}(\Omega) \times L^{1}(\Omega)$ exists and is given by

$$
F_{\alpha, \beta}(u, v):= \begin{cases}\Phi_{\alpha, \beta}(u) & \text { if } v=1 \mathcal{L}^{n} \text {-a.e. in } \Omega \\ +\infty & \text { otherwise. }\end{cases}
$$

Theorem 3.1 directly follows from the estimates for the functionals

$$
F_{\alpha, \beta}^{\prime}:=\Gamma-\liminf _{k \rightarrow \infty} F_{k} \quad \text { and } \quad F_{\alpha, \beta}^{\prime \prime}:=\Gamma-\limsup _{k \rightarrow \infty} F_{k}
$$

stated in the following theorems.

Theorem 3.2. Assume (3.1)-(3.4). Let $(u, v) \in L^{1}(\Omega) \times L^{1}(\Omega)$ be such that $F_{\alpha, \beta}^{\prime}(u, v)$ is finite. Then $v=1 \mathcal{L}^{n}$-a.e. in $\Omega$ and

$$
\Phi_{\alpha, \beta}(u) \leq F_{\alpha, \beta}^{\prime}(u, 1) .
$$

Theorem 3.3. Assume (3.1)-(3.4) and assume that $\Omega$ has Lipschitz boundary. For every $u \in L^{1}(\Omega)$ the following estimate holds

$$
F_{\alpha, \beta}^{\prime \prime}(u, 1) \leq \Phi_{\alpha, \beta}(u) .
$$

Theorem 3.2 is an immediate consequence of the following proposition.

Proposition 3.4. Assume (3.1)-(3.4). Let $\left(u_{k}, v_{k}\right)$ be a sequence in $L^{1}(\Omega) \times L^{1}(\Omega)$ such that

$$
\begin{gathered}
\left(u_{k}, v_{k}\right) \rightarrow(u, v) \text { in } L^{1}(\Omega) \times L^{1}(\Omega), \\
\left(F_{k}\left(u_{k}, v_{k}\right)\right) \text { is bounded. }
\end{gathered}
$$

Then $v=1 \mathcal{L}^{n}$-a.e. in $\Omega$ and

$$
\Phi_{\alpha, \beta}(u) \leq \liminf _{k \rightarrow \infty} \int_{\Omega}\left(v_{k}\left|\nabla u_{k}\right|^{2}+\frac{\psi\left(v_{k}\right)}{\delta_{k}}+c \epsilon_{k}^{p-1}\left|\nabla v_{k}\right|^{p}\right) d x .
$$


Moreover, when $0 \leq \alpha<\infty$ and $0<\beta<\infty$ the following estimates hold

$$
\begin{gathered}
\int_{\Omega}|\nabla u|^{2} d x \leq \liminf _{k \rightarrow \infty} \int_{\Omega} v_{k}\left|\nabla u_{k}\right|^{2} d x \\
a_{\beta} \mathcal{H}^{n-1}\left(J_{u}\right) \leq \liminf _{k \rightarrow \infty} \int_{\Omega}\left(\frac{\psi\left(v_{k}\right)}{\delta_{k}}+c \epsilon_{k}^{p-1}\left|\nabla v_{k}\right|^{p}\right) d x
\end{gathered}
$$

estimate (3.18) also holds if $\alpha=\infty$ or $\beta=0$.

We shall prove the one-dimensional case of Proposition 3.4 and Theorem 3.3 in Section 4, whereas the $n$-dimensional case will be studied in Section 5 .

3.2. The case $\boldsymbol{p}=\infty$. In [10] the limiting case $p=\infty$ when $\beta=1$ is faced. In order to give a complete frame we state now the $\Gamma$-convergence results when $p=\infty$ for different values of $\alpha$ and $\beta$.

Let $\Omega$ be a bounded open subset of $\mathbb{R}^{n}$ and let $\delta_{k}>0, \epsilon_{k} \geq 0, \eta_{k} \geq 0$ be infinitesimal sequences. We define $F_{k}: L^{1}(\Omega) \times L^{1}(\Omega) \rightarrow[0, \infty]$ by

$$
F_{k}(u, v):= \begin{cases}\int_{\Omega}\left(v|\nabla u|^{2}+\frac{\psi(v)}{\delta_{k}}\right) d x & \text { if }(u, v) \in H^{1}(\Omega) \times V_{k} \\ & \text { otherwise }\end{cases}
$$

where,

$$
\begin{gathered}
\psi \in C([0,1]) \text { is strictly decreasing with } \psi(1)=0, \\
V_{k}:=\left\{v \in W^{1, \infty}(\Omega): \eta_{k} \leq v \leq 1,|\nabla v| \leq \frac{1}{\epsilon_{k}} \mathcal{L}^{n} \text {-a.e. in } \Omega\right\} .
\end{gathered}
$$

We assume that the limits

$$
\alpha:=\lim _{k \rightarrow \infty} \frac{\eta_{k}}{\delta_{k}} \quad \text { and } \quad \beta:=\lim _{k \rightarrow \infty} \frac{\delta_{k}}{\epsilon_{k}}
$$

exist. Let $\Phi_{\alpha, \beta}$ be defined as in (3.5)-(3.10) with the only modification that $a_{\beta}$ and $b_{\alpha}$ are now set equal to

$$
a_{\beta}:=\frac{2}{\beta} \int_{0}^{1} \psi d s, \quad b_{\alpha}:=2(\alpha \psi(0))^{\frac{1}{2}} .
$$

Under these hypotheses Theorems 3.1-3.4 holds. For proofs in the case $0<\beta<\infty$ we refer to [10]; in the other regimes proofs are similar to the ones given in next sections for $p<\infty$, with few adaptations.

\section{Proof of the $\Gamma$-Convergence Result in the Case $n=1$.}

Proof of Proposition 3.4. Let $\left(u_{k}, v_{k}\right)$ be a sequence satisfying (3.15) and (3.16) with bounding constant $C$. First we note that (3.16) and (3.2) imply $v=1 \mathcal{L}^{1}$-a.e. in $\Omega$. This in particular concludes the proof in the case $\alpha=0$ and $\beta=\infty$.

Let now $\alpha=\infty$. Up to subsequences we can suppose that the lower limit in the right-hand side of (3.18) is a limit and that $\eta_{k}>0$. We are going to prove that the sequence $\left(\left|\nabla u_{k}\right|\right)$ is equi-integrable. Let $A \subset \Omega$ be a measurable set, then the Hölder Inequality and (3.16) imply

$$
\begin{aligned}
\int_{A}\left|\nabla u_{k}\right| d x & \leq\left(\int_{\Omega} v_{k}\left|\nabla u_{k}\right|^{2} d x\right)^{\frac{1}{2}}\left(\int_{A} 1 / v_{k} d x\right)^{\frac{1}{2}} \\
& \leq C^{\frac{1}{2}}\left(\int_{A \cap\left\{v_{k} \geq 1 / 2\right\}} 1 / v_{k} d x+\int_{A \cap\left\{v_{k}<1 / 2\right\}} 1 / v_{k} d x\right)^{\frac{1}{2}} \\
& \leq C^{\frac{1}{2}}\left(2 \mathcal{L}^{1}(A)+\frac{1}{\psi(1 / 2)} \frac{\delta_{k}}{\eta_{k}} \int_{\Omega} \frac{\psi\left(v_{k}\right)}{\delta_{k}} d x\right)^{\frac{1}{2}} \\
& \leq C^{\frac{1}{2}}\left(2 \mathcal{L}^{1}(A)+\frac{C}{\psi(1 / 2)} \frac{\delta_{k}}{\eta_{k}}\right)^{\frac{1}{2}} .
\end{aligned}
$$


Given $\sigma>0$, the inequality $\frac{C}{\psi(1 / 2)} \frac{\delta_{k}}{\eta_{k}} \leq \frac{\sigma^{2}}{2 C}$ is true for $k$ large since $\alpha=\infty$. Therefore $\mathcal{L}^{1}(A)<\frac{\sigma^{2}}{4 C}$ implies the last term in (4.1) is less than $\sigma$ for $k$ large. Using for the first terms of the sequence the absolute continuity of the Lebesgue integral, we conclude that $\left(\left|\nabla u_{k}\right|\right)$ is equi-integrable. Now the Dunford-Pettis Theorem implies $u \in W^{1,1}(\Omega)$ and $\nabla u_{k} \rightarrow \nabla u$ weakly in $L^{1}(\Omega)$. By a classical lower semicontinuity result (see, for instance, [7, Theorem 2.3.1]) finally we obtain (3.18) and then $u \in H^{1}(\Omega)$.

Let $0 \leq \alpha<\infty$ and $0 \leq \beta<\infty$. In what follows we shall use the notation $I(x, \mu)$ for the interval $(x-\mu, x+\mu)$, whereas we shall write $F_{k}(u, v, I)$ to indicate the functional in (3.1) when the integrals are defined on the set $I$.

Proof of (3.18). Let $x_{0} \in \Omega$ and $\mu>0$ be such that $u$ is absolutely continuous in $I\left(x_{0}, \mu\right) \subset \Omega$. Now the same argument used by Ambrosio and Tortorelli in [3, Lemma 4.2] and [4, Lemma 2.1] works here with obvious adaptations. We conclude that $u \in H^{1}\left(I\left(x_{0}, \mu\right)\right)$ and $(3.18)$ holds in $I\left(x_{0}, \mu\right)$.

Proof of (3.19). Let now $x_{0}$ be a point such that $u$ is not absolutely continuous in any interval of the form $I\left(x_{0}, \mu\right)$. We sketch the argument proposed by Ambrosio and Tortorelli in [4, Lemma 2.1] in order to prove that there are only finitely many points of such a type.

Let $\mu>0$ small enough; since $u$ is not absolutely continuous in $I\left(x_{0}, \mu / 2\right)$, the infimum $\inf _{I\left(x_{0}, \mu / 2\right)} v_{k}$ tends to 0 and this guarantees the existence for every $k$ of a point $x_{0}-\mu / 2<x_{k}<x_{0}+\mu / 2$ such that $v_{k}\left(x_{k}\right) \rightarrow 0$. Moreover, up to subsequences, $v_{k} \rightarrow 1 \mathcal{L}^{1}$-a.e. in $\Omega$, so that we can find two points $x_{0}-\mu<y_{1}<x_{k}<y_{2}<x_{0}+\mu$ with $v_{k}\left(y_{1}\right) \rightarrow 1$ and $v_{k}\left(y_{2}\right) \rightarrow 1$. The Young Inequality now gives

$$
\begin{aligned}
F_{k}\left(u_{k}, v_{k}, I\left(x_{0}, \mu\right)\right) & \geq(c p)^{\frac{1}{p}}\left(\frac{q \epsilon_{k}}{\delta_{k}}\right)^{\frac{1}{q}} \int_{y_{1}}^{x_{k}} \psi\left(v_{k}\right)^{\frac{1}{q}}\left|\nabla v_{k}\right| d x+(c p)^{\frac{1}{p}}\left(\frac{q \epsilon_{k}}{\delta_{k}}\right)^{\frac{1}{q}} \int_{x_{k}}^{y_{2}} \psi\left(v_{k}\right)^{\frac{1}{q}}\left|\nabla v_{k}\right| d x \\
& \geq(c p)^{\frac{1}{p}}\left(\frac{q \epsilon_{k}}{\delta_{k}}\right)^{\frac{1}{q}} \int_{v_{k}\left(x_{k}\right)}^{v_{k}\left(y_{1}\right)} \psi(s)^{\frac{1}{q}} d s+(c p)^{\frac{1}{p}}\left(\frac{q \epsilon_{k}}{\delta_{k}}\right)^{\frac{1}{q}} \int_{v_{k}\left(x_{k}\right)}^{v_{k}\left(y_{2}\right)} \psi(s)^{\frac{1}{q}} d s .
\end{aligned}
$$

Passing to the lower limit in the previous inequality we obtain

$$
\liminf _{k \rightarrow \infty} F_{k}\left(u_{k}, v_{k}, I\left(x_{0}, \mu\right)\right) \geq a_{\beta}>0
$$

in the case $0<\beta<\infty$. Since the left-hand side in (4.3) is bounded by (3.16), the number of disjoint intervals such as $I\left(x_{0}, \mu\right)$ is bounded by a constant independent by $\mu$. This implies $u \in S B V(\Omega)$ and (3.19) follows. From (3.18) we also deduce $u \in S B V^{2}(\Omega)$.

In the case $\beta=0$ we achieve a contradiction since the left-hand side of (4.2) is bounded by (3.16), whereas the right-hand side tends to infinity. Therefore, each point of $\Omega$ satisfies the previous step, so that $u \in H^{1}(\Omega)$ and (3.18) holds.

Proof of (3.17) in the case $0<\beta<\infty$. First we note that (3.18) and (3.19) lead to (3.17) in the case $\alpha=0,0<\beta<\infty$.

It remains to consider the case $0<\alpha<\infty, 0<\beta<\infty$. We shall define suitably six points in place of $y_{1}, x_{k}, y_{2}$; in this way we determine some intervals we shall study separately. In the external intervals, we shall be able to repeat the previous argument by Ambrosio and Tortorelli, the two in-between intervals will be neglected, and the central one will give rise to the cohesive term.

Let $x_{0} \in J_{u}$ and assume $u^{-}\left(x_{0}\right)<u^{+}\left(x_{0}\right)$. Let $0<\sigma<\left|\left[u\left(x_{0}\right)\right]\right| / 2$ and let $\mu>0$ be such that $\left|u(x)-u^{ \pm}\left(x_{0}\right)\right|<\sigma / 2$ for $0<\left|x-x_{0}\right| \leq \mu / 2$; since $u_{k} \rightarrow u \mathcal{L}^{1}$-a.e. in $\Omega$ up to subsequences, it is not restrictive to assume $u_{k}\left(x_{0} \pm \mu / 2\right) \rightarrow u\left(x_{0} \pm \mu / 2\right)$. We prove that there exist six points $y_{1}<x_{k}^{1} \leq \tilde{x}_{k}^{1}<\tilde{x}_{k}^{2} \leq x_{k}^{2}<y_{2}$ in the interval $I\left(x_{0}, \mu\right)$, such that

$$
\begin{gathered}
\lim _{k \rightarrow \infty} v_{k}\left(y_{1}\right)=\lim _{k \rightarrow \infty} v_{k}\left(y_{2}\right)=1, \\
\lim _{k \rightarrow \infty} v_{k}\left(x_{k}^{1}\right)=\lim _{k \rightarrow \infty} v_{k}\left(x_{k}^{2}\right)=0, \\
u_{k}\left(\tilde{x}_{k}^{1}\right)=u^{-}\left(x_{0}\right)+\sigma, \quad u_{k}\left(\tilde{x}_{k}^{2}\right)=u^{+}\left(x_{0}\right)-\sigma .
\end{gathered}
$$

Let us define

$$
\tilde{x}_{k}^{1}:=\max \left\{x \in\left[x_{0}-\mu / 2, x_{0}+\mu / 2\right]: u_{k}(x) \leq u^{-}\left(x_{0}\right)+\sigma\right\} .
$$


Since $\left|u_{k}\left(x_{0} \pm \mu / 2\right)-u^{ \pm}\left(x_{0}\right)\right|<\sigma$ for $k$ large, the continuity of $u_{k}$ implies that $\tilde{x}_{k}^{1}$ is well-defined, that $\tilde{x}_{k}^{1}<x_{0}+\mu / 2$, and that $u_{k}\left(\tilde{x}_{k}^{1}\right)=u^{-}\left(x_{0}\right)+\sigma$.

We now verify that $x_{0} \leq \liminf _{k \rightarrow \infty} \tilde{x}_{k}^{1}$. If not up to subsequences we have $\tilde{x}_{k}^{1}<c_{0}<x_{0}$, where $c_{0}$ is a constant. Using the definition of $\tilde{x}_{k}^{1}$ we obtain, as $k \rightarrow \infty$, that $u(x) \geq u^{-}\left(x_{0}\right)+\sigma$ in $\left(c_{0}, x_{0}\right)$. As $x \rightarrow x_{0}^{-}$we get a contradiction.

We claim now that

$$
\limsup _{k \rightarrow \infty} \inf _{\left[x_{0}-\mu / 2, \tilde{x}_{k}^{1}\right]} v_{k} \leq 0 .
$$

By contradiction we assume that the opposite inequality holds. By this and (3.16) we have, up to subsequences, that

$$
\int_{x_{0}-\mu / 2}^{\tilde{x}_{k}^{1}}\left|\nabla u_{k}\right|^{2} d x \quad \text { is bounded. }
$$

Let us verify now that $\lim \sup _{k \rightarrow \infty} \tilde{x}_{k}^{1} \leq x_{0}$. We argue again by contradiction and suppose $\tilde{x}_{k}^{1}>c_{1}>x_{0}$, where $c_{1}$ is a constant. Up to subsequences the integral $\int_{x_{0}-\mu / 2}^{c_{1}}\left|\nabla u_{k}\right|^{2} d x$ is bounded by (4.5), so that $u$ is continuous in $x_{0}$ and this contradicts the assumption $x_{0} \in J_{u}$. Therefore we conclude $\tilde{x}_{k}^{1} \rightarrow x_{0}$.

Now, by the absolute continuity of $u_{k}$ and the Hölder Inequality we obtain for every $y \in\left(x_{0}-\right.$ $\left.\mu / 2, \tilde{x}_{k}^{1}\right)$

$$
\left|u_{k}\left(\tilde{x}_{k}^{1}\right)-u_{k}(y)\right| \leq\left|\tilde{x}_{k}^{1}-y\right|^{\frac{1}{2}}\left(\int_{y}^{\tilde{x}_{k}^{1}}\left|\nabla u_{k}\right|^{2} d x\right)^{\frac{1}{2}} \leq c_{2}\left|\tilde{x}_{k}^{1}-y\right|^{\frac{1}{2}},
$$

where in the last inequality $c_{2}<\infty$ is a constant and we have used (4.5). Let us fix $y \in\left(x_{0}-\mu, x_{0}\right)$ such that $u_{k}(y) \rightarrow u(y)$; then $y \in\left(x_{0}-\mu, \tilde{x}_{k}^{1}\right)$ for $k$ large, so that inequality

$$
\left|u^{-}\left(x_{0}\right)+\sigma-u_{k}(y)\right| \leq c_{2}\left|\tilde{x}_{k}^{1}-y\right|^{\frac{1}{2}}
$$

follows from $u_{k}\left(\tilde{x}_{k}^{1}\right)=u^{-}\left(x_{0}\right)+\sigma$ and (4.6). Passing to the limit first as $k \rightarrow \infty$ and then as $y \rightarrow x_{0}^{-}$ we achieve a contradiction and the claim (4.4) is proved.

By (4.4) we are able to find a sequence $x_{0}-\mu / 2 \leq x_{k}^{1} \leq \tilde{x}_{k}^{1}$ such that $v_{k}\left(x_{k}^{1}\right) \rightarrow 0$. Since $v_{k} \rightarrow 1$ $\mathcal{L}^{1}$-a.e. in $\Omega$, we also find a point $y_{1} \in\left(x_{0}-\mu, x_{0}-\mu / 2\right)$ such that $v_{k}\left(y_{1}\right) \rightarrow 1$.

Let us define now

$$
\tilde{x}_{k}^{2}:=\min \left\{x \in\left[\tilde{x}_{k}^{1}, x_{0}+\mu / 2\right]: u_{k}(x) \geq u^{+}\left(x_{0}\right)-\sigma\right\} .
$$

We can easily prove that it is well-defined, that $u_{k}\left(\tilde{x}_{k}^{2}\right)=u^{+}\left(x_{0}\right)-\sigma$, and that $\tilde{x}_{k}^{2} \rightarrow x_{0}$. Note that the convergence $\tilde{x}_{k}^{2} \rightarrow x_{0}$ implies the convergence $\tilde{x}_{k}^{1} \rightarrow x_{0}$.

As before we can also prove that

$$
\limsup _{k \rightarrow \infty} \inf _{\left[\tilde{x}_{k}^{2}, x_{0}+\mu / 2\right]} v_{k}=0
$$

and the existence of $x_{k}^{2}$ and $y_{2}$ follows.

Now let us proceed with the computation. In the intervals $\left(y_{1}, x_{k}^{1}\right)$ and $\left(x_{k}^{2}, y_{2}\right)$ we can repeat the argument by Ambrosio and Tortorelli in (4.3), so that

$$
\liminf _{k \rightarrow \infty} F_{k}\left(u_{k}, v_{k},\left(y_{1}, x_{k}^{1}\right) \cup\left(x_{k}^{2}, y_{2}\right)\right) \geq a_{\beta} .
$$

It remains to estimate the functional in the interval $I_{k}:=\left(\tilde{x}_{k}^{1}, \tilde{x}_{k}^{2}\right)$. Let us define

$$
\begin{gathered}
W_{k}:=\left\{w \in H^{1}\left(I_{k}\right), w\left(\tilde{x}_{k}^{1}\right)=u^{-}\left(x_{0}\right)+\sigma, w\left(\tilde{x}_{k}^{2}\right)=u^{+}\left(x_{0}\right)-\sigma\right\}, \\
Z_{k}:=\left\{z \in W^{1, p}\left(I_{k}\right), \eta_{k} \leq z \leq 1 \mathcal{L}^{1} \text {-a.e. on } I_{k}\right\} \\
H_{k}(w, z):=\int_{I_{k}}\left(z|\nabla w|^{2}+\frac{\psi(z)}{\delta_{k}}\right) d x, \quad \text { for }(w, z) \in W_{k} \times Z_{k}, \\
h_{k}(z):=\min _{w \in W_{k}} H_{k}(w, z), \quad \text { for } z \in Z_{k} .
\end{gathered}
$$


By elementary computation we find that this minimum is achieved and that

$$
h_{k}(z)=\frac{\left(\left[u\left(x_{0}\right)\right]-2 \sigma\right)^{2}}{\int_{I_{k}} \frac{1}{z} d x}+\int_{I_{k}} \frac{\psi(z)}{\delta_{k}} d x
$$

Let now $0<\lambda<1$. We observe that

$$
\begin{gathered}
\int_{\left\{x \in I_{k}: v_{k} \geq \lambda\right\}} \frac{1}{v_{k}} d x \leq \frac{\mathcal{L}^{1}\left(I_{k}\right)}{\lambda} \\
\int_{\left\{x \in I_{k}: v_{k}<\lambda\right\}} \frac{1}{v_{k}} d x \leq \frac{1}{\psi(\lambda)} \frac{\delta_{k}}{\eta_{k}}\left(\int_{I_{k}} \frac{\psi\left(v_{k}\right)}{\delta_{k}} d x\right) .
\end{gathered}
$$

We use the previous inequalities to estimate the functional $F_{k}\left(u_{k}, v_{k}, I_{k}\right)$ :

$$
\begin{aligned}
F_{k}\left(u_{k}, v_{k}, I_{k}\right) & \geq H_{k}\left(u_{k}, v_{k}\right) \\
& \geq h_{k}\left(v_{k}\right) \\
& \geq \frac{\left(\left[u\left(x_{0}\right)\right]-2 \sigma\right)^{2}}{\frac{\mathcal{L}^{1}\left(I_{k}\right)}{\lambda}+\frac{1}{\psi(\lambda)} \frac{\delta_{k}}{\eta_{k}}\left(\int_{I_{k}} \frac{\psi\left(v_{k}\right)}{\delta_{k}} d x\right)}+\int_{I_{k}} \frac{\psi\left(v_{k}\right)}{\delta_{k}} d x \\
& \geq 2\left(\frac{\eta_{k} \psi(\lambda)}{\delta_{k}}\right)^{\frac{1}{2}}\left(\left[u\left(x_{0}\right)\right]-2 \sigma\right)-\frac{\eta_{k} \psi(\lambda) \mathcal{L}^{1}\left(I_{k}\right)}{\lambda \delta_{k}}
\end{aligned}
$$

where to get the last inequality we have minimized in $[0, \infty[$ the function

$$
t \mapsto \frac{\left(\left[u\left(x_{0}\right)\right]-2 \sigma\right)^{2}}{\frac{\mathcal{L}^{1}\left(I_{k}\right)}{\lambda}+\frac{1}{\psi(\lambda)} \frac{\delta_{k}}{\eta_{k}} t}+t
$$

Passing to the limit first as $k \rightarrow \infty$, then as $\lambda \rightarrow 0$, and finally as $\sigma \rightarrow 0$ we obtain

$$
\liminf _{k \rightarrow \infty} F_{k}\left(u_{k}, v_{k}, I_{k}\right) \geq b_{\alpha}\left|\left[u\left(x_{0}\right)\right]\right|
$$

Inequalities (3.18) for the set $I\left(x_{0}, \mu\right),(4.7)$, and (4.9) lead to (3.17).

It remains to study the case $0<\alpha<\infty, \beta=\infty$. By [6, Theorem 2.1] the functional $\Phi_{\alpha, \infty}$ is weakly* lower semicontinuous in $B V(\Omega)$ and strongly lower semicontinuous in $L^{1}(\Omega)$, so that it is sufficient to prove that

$$
\liminf _{k \rightarrow \infty} \Phi_{\alpha, \infty}\left(u_{k}\right) \leq \liminf _{k \rightarrow \infty} F_{k}\left(u_{k}, v_{k}\right)
$$

In order to simplify the notation we set $A_{k}:=\left\{\left|\nabla u_{k}\right|<b_{\alpha} / 2\right\}$; we compute the integrals of $f_{\alpha}\left(\left|\nabla u_{k}\right|\right)$ on $A_{k}$ and on $A_{k}^{c}$.

Let us fix $0<\lambda<\mu<1$. First we note that the convergence in measure $v_{k} \rightarrow 1$ implies

$$
\int_{A_{k} \cap\left\{v_{k}<\mu\right\}} f_{\alpha}\left(\left|\nabla u_{k}\right|\right) d x+\int_{A_{k} \cap\left\{v_{k} \geq \mu\right\}} f_{\alpha}\left(\left|\nabla u_{k}\right|\right) d x \leq \int_{A_{k} \cap\left\{v_{k} \geq \mu\right\}} f_{\alpha}\left(\left|\nabla u_{k}\right|\right) d x+o(1) .
$$


On $A_{k}^{c}$ we have

$$
\begin{aligned}
& \int_{A_{k}^{c} \cap\left\{v_{k} \geq \mu\right\}} f_{\alpha}\left(\left|\nabla u_{k}\right|\right) d x+\int_{A_{k}^{c} \cap\left\{\lambda<v_{k}<\mu\right\}} f_{\alpha}\left(\left|\nabla u_{k}\right|\right) d x+\int_{A_{k}^{c} \cap\left\{v_{k} \leq \lambda\right\}} f_{\alpha}\left(\left|\nabla u_{k}\right|\right) d x \leq \\
\leq & \int_{A_{k}^{c} \cap\left\{v_{k} \geq \mu\right\}}\left|\nabla u_{k}\right|^{2} d x+\int_{A_{k}^{c} \cap\left\{\lambda<v_{k}<\mu\right\}} b_{\alpha}\left(\left|\nabla u_{k}\right|-\frac{b_{\alpha}}{4}\right) d x+\int_{A_{k}^{c} \cap\left\{v_{k} \leq \lambda\right\}} b_{\alpha}\left(\left|\nabla u_{k}\right|-\frac{b_{\alpha}}{4}\right) d x \\
\leq & \int_{A_{k}^{c} \cap\left\{v_{k} \geq \mu\right\}}\left|\nabla u_{k}\right|^{2} d x+b_{\alpha}\left(\int_{\Omega} v_{k}\left|\nabla u_{k}\right|^{2} d x\right)^{\frac{1}{2}}\left(\int_{\left\{\lambda<v_{k}<\mu\right\}} \frac{1}{v_{k}} d x\right)^{\frac{1}{2}} \\
& +b_{\alpha}\left(\int_{\left\{v_{k} \leq \lambda\right\}} v_{k}\left|\nabla u_{k}\right|^{2} d x\right)^{\frac{1}{2}}\left(\int_{\left\{v_{k} \leq \lambda\right\}} \frac{1}{v_{k}} d x\right)^{\frac{1}{2}} \\
\leq & \int_{A_{k}^{c} \cap\left\{v_{k} \geq \mu\right\}}\left|\nabla u_{k}\right|^{2} d x+b_{\alpha}\left(\frac{C}{\lambda}\right)^{\frac{1}{2}} \mathcal{L}^{1}\left(\left\{v_{k}<\mu\right\}\right)^{\frac{1}{2}} \\
& +b_{\alpha}\left(\frac{1}{\psi(\lambda)} \frac{\delta_{k}}{\eta_{k}}\right)^{\frac{1}{2}}\left(\int_{\left\{v_{k} \leq \lambda\right\}} v_{k}\left|\nabla u_{k}\right|^{2} d x\right)^{\frac{1}{2}}\left(\int_{\left\{v_{k} \leq \lambda\right\}} \frac{\psi\left(v_{k}\right)}{\delta_{k}} d x\right)^{\frac{1}{2}} \\
\leq & \int_{A_{k}^{c} \cap\left\{v_{k} \geq \mu\right\}}\left|\nabla u_{k}\right|^{2} d x+\frac{b_{\alpha}}{2}\left(\frac{1}{\psi(\lambda)} \frac{\delta_{k}}{\eta_{k}}\right)^{\frac{1}{2}} \int_{\left\{v_{k} \leq \lambda\right\}}\left(v_{k}\left|\nabla u_{k}\right|^{2}+\frac{\psi\left(v_{k}\right)}{\delta_{k}}\right) d x+o(1)
\end{aligned}
$$

where in the first inequality we have used the definition of $f_{\alpha}$ and the fact that $b_{\alpha}\left(t-b_{\alpha} / 4\right) \leq t^{2}$ for $t \geq b_{\alpha} / 2$; the Hölder Inequality justifies the second estimate; the property (3.16) and an easy computation have led to the third inequality; finally from the Cauchy Inequality and the convergence in measure $v_{k} \rightarrow 1$ we have found the final expression. From (4.11) and (4.12) we deduce

$$
\begin{aligned}
\Phi_{\alpha, \infty}\left(u_{k}\right) \leq & \int_{\left\{v_{k} \geq \mu\right\}}\left|\nabla u_{k}\right|^{2} d x+\frac{b_{\alpha}}{2}\left(\frac{1}{\psi(\lambda)} \frac{\delta_{k}}{\eta_{k}}\right)^{\frac{1}{2}} \int_{\left\{v_{k} \leq \lambda\right\}}\left(v_{k}\left|\nabla u_{k}\right|^{2}+\frac{\psi\left(v_{k}\right)}{\delta_{k}}\right) d x+o(1) \\
\leq & \frac{1}{\mu} \int_{\left\{v_{k} \geq \mu\right\}}\left(v_{k}\left|\nabla u_{k}\right|^{2}+\frac{\psi\left(v_{k}\right)}{\delta_{k}}\right) d x \\
& +\frac{b_{\alpha}}{2}\left(\frac{1}{\psi(\lambda)} \frac{\delta_{k}}{\eta_{k}}\right)^{\frac{1}{2}} \int_{\left\{v_{k} \leq \lambda\right\}}\left(v_{k}\left|\nabla u_{k}\right|^{2}+\frac{\psi\left(v_{k}\right)}{\delta_{k}}\right) d x+o(1) \\
\leq & \max \left(\frac{1}{\mu}, \frac{b_{\alpha}}{2}\left(\frac{1}{\psi(\lambda)} \frac{\delta_{k}}{\eta_{k}}\right)^{\frac{1}{2}}\right) F_{k}\left(u_{k}, v_{k}\right)+o(1) .
\end{aligned}
$$

Passing to the limit first as $k \rightarrow \infty$ and then as $\lambda \rightarrow 0, \mu \rightarrow 1$ we obtain (4.10).

Let us complete the one-dimensional case of the $\Gamma$-convergence result by proving the upper estimate.

Proof of Theorem 3.3. The cases $\alpha=\infty$ or $\beta=0$ are trivial since $F_{\alpha, \beta}^{\prime}(u, v)<\infty$ implies $u \in H^{1}(\Omega)$ and $v=1 \mathcal{L}^{1}$-a.e. in $\Omega$.

Let now $0 \leq \alpha<\infty$ and $0<\beta<\infty$ and let $u$ be such that $\Phi_{\alpha, \beta}(u, 1)<\infty$. A truncation argument shows that in dimension $n=1$ a function $u \in G S B V^{2}(\Omega) \cap L^{1}(\Omega)$ with $\Phi_{0, \beta}(u, 1)<\infty$ actually belongs to $S B V^{2}(\Omega)$. Therefore, both for $\alpha=0$ and for $0<\alpha<\infty$, we start with a function $u \in S B V^{2}(\Omega)$; for simplicity we also suppose $J_{u}=\{\bar{x}\}$. Let $\left(\sigma_{k}^{\alpha}\right)$ and $\left(\mu_{k}\right)$ be positive infinitesimal sequences which we shall specify later and let

$$
A_{k}:=\left(\bar{x}-\sigma_{k}^{\alpha}, \bar{x}+\sigma_{k}^{\alpha}\right) \quad \text { and } \quad B_{k}:=\left(\bar{x}-\sigma_{k}^{\alpha}-\mu_{k}, \bar{x}-\sigma_{k}^{\alpha}\right) \cup\left(\bar{x}+\sigma_{k}^{\alpha}, \bar{x}+\sigma_{k}^{\alpha}+\mu_{k}\right) .
$$

Let us define $u_{k}$ by $u$ out of $A_{k}$ and linking linearly in $A_{k}$.

$$
\text { Let } f(\rho):=\psi(1-\rho), g(\rho):=\frac{1}{\int_{0}^{1-\rho} \psi^{-\frac{1}{p}} d s} \text {, and } h:=(f g)^{\frac{1}{2}} \text { for } 0<\rho<1 \text {; we note that } h \text { is }
$$
strictly increasing and that $h$ and $f / g$ are infinitesimal in 0 . Then the sequence $\rho_{k}:=h^{-1}\left(\delta_{k}\right)$ is infinitesimal and

$$
\frac{f\left(\rho_{k}\right)}{\delta_{k}} \rightarrow 0, \quad \frac{\delta_{k}}{g\left(\rho_{k}\right)} \rightarrow 0
$$


We now set $v_{k}$ equal to $\eta_{k}$ in $A_{k}$ and equal to $1-\rho_{k}$ out of $A_{k} \cup B_{k}$.

In order to define $v_{k}$ everywhere, we first consider the following Cauchy problem

$$
\left\{\begin{array}{l}
w_{k}^{\prime}=\left(\frac{q}{c p \delta_{k}}\right)^{\frac{1}{p}} \epsilon_{k}^{-\frac{1}{q}} \psi\left(w_{k}\right)^{\frac{1}{p}} \\
w_{k}(0)=\eta_{k} .
\end{array}\right.
$$

Since $\eta_{k}<1$ and $\psi$ is continuous and strictly positive in $[0,1)$, the previous problem has only one solution $w_{k}$ in the interval $\left[0, T_{k}\right)$, where $T_{k} \in(0, \infty]$ is defined by

$$
T_{k}:=\left(\frac{c p \delta_{k}}{q}\right)^{\frac{1}{p}} \epsilon_{k}^{\frac{1}{q}} \int_{\eta_{k}}^{1} \psi^{-\frac{1}{p}} d s .
$$

Precisely, the solution $w_{k}$ is obtained by taking the inverse of the function

$$
z \in\left[\eta_{k}, 1\right) \mapsto\left(\frac{c p \delta_{k}}{q}\right)^{\frac{1}{p}} \epsilon_{k}^{\frac{1}{q}} \int_{\eta_{k}}^{z} \psi^{-\frac{1}{p}} d s \in\left[0, T_{k}\right) .
$$

By this we can define

$$
\begin{gathered}
v_{k}(x):=w_{k}\left(|x-\bar{x}|-\sigma_{k}^{\alpha}\right) \text { on } B_{k}, \\
\mu_{k}:=\left(\frac{c p \delta_{k}}{q}\right)^{\frac{1}{p}} \epsilon_{k}^{\frac{1}{q}} \int_{\eta_{k}}^{1-\rho_{k}} \psi^{-\frac{1}{p}} d s
\end{gathered}
$$

where $\mu_{k}$ is infinitesimal by $(4.13)$.

Then $\left(u_{k}, v_{k}\right) \in H^{1}(\Omega) \times V_{\eta_{k}}$ and $\left(u_{k}, v_{k}\right) \rightarrow(u, 1)$ in $L^{1}(\Omega) \times L^{1}(\Omega)$. An easy computation shows that

$$
\begin{gathered}
\int_{\Omega \backslash A_{k}} v_{k}\left|\nabla u_{k}\right|^{2} d x \leq \int_{\Omega}|\nabla u|^{2} d x \\
\int_{\Omega \backslash\left(A_{k} \cup B_{k}\right)} \frac{\psi\left(v_{k}\right)}{\delta_{k}} d x \leq \frac{\psi\left(1-\rho_{k}\right)}{\delta_{k}} \mathcal{L}^{1}(\Omega), \\
\int_{A_{k}}\left(v_{k}\left|\nabla u_{k}\right|^{2}+\frac{\psi\left(v_{k}\right)}{\delta_{k}}\right) d x=\frac{\eta_{k}}{2 \sigma_{k}^{\alpha}}\left(u\left(\bar{x}+\sigma_{k}^{\alpha}\right)-u\left(\bar{x}-\sigma_{k}^{\alpha}\right)\right)^{2}+2 \psi\left(\eta_{k}\right) \frac{\sigma_{k}^{\alpha}}{\delta_{k}} .
\end{gathered}
$$

We note that the integral in (4.18) tends to 0 by (4.13). If $\alpha=0$ we take $\sigma_{k}^{0}$ such that $\eta_{k} / \sigma_{k}^{0} \rightarrow 0$ and $\sigma_{k}^{0} / \delta_{k} \rightarrow 0$; by this choice the integral in (4.19) converges to 0 . Whereas if $0<\alpha<\infty$ we define $\sigma_{k}^{\alpha}:=\frac{1}{2}\left(\frac{\alpha}{\psi(0)}\right)^{\frac{1}{2}}|[u(\bar{x})]| \delta_{k}$ and the integral in (4.19) tends to $b_{\alpha}|[u(\bar{x})]|$.

Let us compute now the integral on $B_{k}$. Thanks to the choice of $w_{k}$ the Young Inequality holds with equality, so that

$$
\begin{aligned}
\int_{B_{k}}\left(\frac{\psi\left(v_{k}\right)}{\delta_{k}}+c \epsilon_{k}^{p-1}\left|\nabla v_{k}\right|^{p}\right) d x & =2(c p)^{\frac{1}{p}}\left(\frac{q \epsilon_{k}}{\delta_{k}}\right)^{\frac{1}{q}} \int_{0}^{\mu_{k}} \psi\left(w_{k}\right)^{\frac{1}{q}} w_{k}^{\prime} d x \\
& =2(c p)^{\frac{1}{p}}\left(\frac{q \epsilon_{k}}{\delta_{k}}\right)^{\frac{1}{q}} \int_{\eta_{k}}^{1-\rho_{k}} \psi(s)^{\frac{1}{q}} d s .
\end{aligned}
$$

As $k \rightarrow \infty$ this term tends to $a_{\beta}$ and the proof is complete.

Let us consider now the case $\alpha=0, \beta=\infty$. First we suppose that $u$ is piecewise constant with $J_{u}=\{\bar{x}\}$. If this is the case we define all parameters as before, so that by repeating the computations in (4.17)-(4.20) we obtain that $F_{0, \infty}^{\prime \prime}(u, 1)$ is null. In the general case when $u \in L^{1}(\Omega)$ we argue by approximation with piecewise constant functions; since $F_{0, \infty}^{\prime \prime}$ is lower semicontinuous we achieve the same conclusion as before.

The last case to study is $0<\alpha<\infty, \beta=\infty$. By [6, Theorem 3.1] if we prove that for every $u \in S B V^{2}(\Omega)$ we have

$$
F_{\alpha, \infty}^{\prime \prime}(u, 1) \leq \int_{\Omega}|\nabla u|^{2} d x+b_{\alpha} \int_{J_{u}}|[u]| d \mathcal{H}^{0}
$$


we are done, since the left-hand side is lower semicontinuous in $L^{1}(\Omega)$ and the lower semicontinuous envelope of the right-hand side is $\Phi_{\alpha, \infty}$. Inequality (4.21) is easily proved by defining all parameters as before and repeating the computation in (4.17)-(4.20).

\section{Proof of the $\Gamma$-Convergence Result in the Case $n>1$}

Proof of Proposition 3.4. The case $0 \leq \alpha<\infty, \beta=\infty$ and the case $\alpha=\infty$ can be faced as for $n=1$. In all other regimes we can argue by slicing and the proof of [10, Proposition 1] works here with obvious modifications.

Let us focus now on the $\Gamma$-limsup inequality.

Proof of Theorem 3.3. The cases $\alpha=\infty$ or $\beta=0$ are trivial.

Let now $0 \leq \alpha<\infty$ and $0<\beta<\infty$. Let $u \in G S B V^{2}(\Omega) \cap L^{1}(\Omega)$ and we consider first the case $u \in L^{\infty}(\Omega)$, so that $u$ belongs in effect to $S B V^{2}(\Omega) \cap L^{\infty}(\Omega)$.

The first part of the proof is the same given for [10, Theorem 3.3], so that we sketch the main ideas. First we need an approximation result in order to work with more regular objects. To this aim we use a corollary of [8, Theorem 3.1] which is stated in [10, Theorem 6.1]. Thanks to [10, Theorem 6.1] it is enough to prove (3.14) for a cube $Q$ and for a function $u \in S B V^{2}(\Omega) \cap L^{\infty}(\Omega)$ such that

$$
\mathcal{H}^{n-1}\left(S \backslash J_{u}\right)=0, \quad u \in W^{k, \infty}(Q \backslash S) \text { for every } k,
$$

where $S=\cup_{i=1}^{m} S_{i}$ with $S_{i}$ closed pairwise disjoint $(n-1)$-simplexes contained in $Q$. To simplify the exposition we consider only the case $m=1$, so that $S$ is a $(n-1)$-simplex; in addition we assume that $S \subset\left\{x_{n}=0\right\}$. We write a point $x \in \mathbb{R}^{n}$ as $x=\left(\bar{x}, x_{n}\right) \in \mathbb{R}^{n-1} \times \mathbb{R}$ and we orient $J_{u}$ so that the normal vector $\nu_{u}$ coincides with $(0,1)$. Let $\Omega^{ \pm}:=\left\{x \in \Omega: \pm x_{n}>0\right\}$ and let $L$ be the maximum between the Lipschitz constants of $u$ in $\Omega^{+}$and $\Omega^{-}$.

Let us define $\sigma_{k}^{\alpha}(\bar{x}):=\frac{1}{2} \delta_{k}\left(\frac{\alpha}{\psi(0)}\right)^{1 / 2}|[u(\bar{x}, 0)]|$ for $x=\left(\bar{x}, x_{n}\right) \in \Omega$ in the case $0<\alpha<\infty$; whereas for $\alpha=0$ we define $\sigma_{k}^{0}$ as any sequence of constant functions such that $\eta_{k} / \sigma_{k}^{0} \rightarrow 0$ and $\sigma_{k}^{0} / \delta_{k} \rightarrow 0$. We observe that $\sigma_{k}^{\alpha}$ is Lipschitz since $u^{+}$and $u^{-}$are; moreover in the case $0<\alpha<\infty$ we have $\sigma_{k}^{\alpha}(\bar{x})=0$ for $\bar{x} \in \partial S$, where $\partial S$ is the boundary of $S$ in the relative topology of $\mathbb{R}^{n-1} \times\{0\}$.

Let

$$
\begin{gathered}
A_{k}:=\left\{x \in \mathbb{R}^{n}:(\bar{x}, 0) \in S,\left|x_{n}\right|<\sigma_{k}^{\alpha}(\bar{x})\right\}, \\
A_{k}^{\prime}:=\left\{x \in \mathbb{R}^{n}:(\bar{x}, 0) \notin S, d(x, \partial S)<\sigma_{k}^{\alpha}(\bar{x})\right\},
\end{gathered}
$$

where $d(x, S)$ is the distance from the point $x$ to the set $S$. The closure of $A_{k} \cup A_{k}^{\prime}$ is contained in $\Omega$ for $k$ large.

Let

$$
u_{k}\left(\bar{x}, x_{n}\right):= \begin{cases}\frac{x_{n}+\sigma_{k}^{\alpha}}{2 \sigma_{k}^{\alpha}}\left(u\left(\bar{x}, \sigma_{k}^{\alpha}\right)-u\left(\bar{x},-\sigma_{k}^{\alpha}\right)\right)+u\left(\bar{x},-\sigma_{k}^{\alpha}\right) & \text { if } x \in A_{k}, \\ u(x) & \text { if } x \in \Omega \backslash\left(A_{k} \cup A_{k}^{\prime}\right) .\end{cases}
$$

In the case $0<\alpha<\infty$ we have $A_{k}^{\prime}=\varnothing$ and then $u_{k} \in W^{1, \infty}(\Omega)$; whereas for $\alpha=0$ the function $u_{k}$ is Lipschitz continuous in $\Omega \backslash A_{k}^{\prime}$ with constant $4 M / \delta_{k}^{0}+12 n L$, so that the McShane Theorem implies the existence of a function, still denoted by $u_{k}$, which extends $u_{k}$ to $A_{k}^{\prime}$ and has the same Lipschitz constant as $u_{k}$ (see [10, Theorem 3.3] for more details). From the definition of $u_{k}$ we immediately deduce that $u_{k} \rightarrow u$ in $L^{1}(\Omega)$. 
Let now $\rho_{k}, w_{k}$ and $\mu_{k}$ be defined as in the one-dimensional case by (4.13), (4.14), and (4.16); we are able to define now

$$
\begin{gathered}
B_{k}:=\left\{x \in \mathbb{R}^{n}:(\bar{x}, 0) \in S, 0 \leq\left|x_{n}\right|-\sigma_{k}^{\alpha}(\bar{x}) \leq \mu_{k}\right\}, \\
B_{k}^{\prime}:=\left\{x \in \mathbb{R}^{n}:(\bar{x}, 0) \notin S, 0 \leq d(x, \partial S)-\sigma_{k}^{\alpha}(\bar{x}) \leq \mu_{k}\right\},
\end{gathered}
$$

and

$$
v_{k}(x):= \begin{cases}\eta_{k} & \text { if } x \in A_{k} \cup A_{k}^{\prime}, \\ w_{k}\left(\left|x_{n}\right|-\sigma_{k}^{\alpha}(\bar{x})\right) & \text { if } x \in B_{k}, \\ w_{k}\left(d(x, \partial S)-\sigma_{k}^{\alpha}(\bar{x})\right) & \text { if } x \in B_{k}^{\prime}, \\ 1-\rho_{k} & \text { otherwise. }\end{cases}
$$

By this choice $\eta_{k} \leq v_{k} \leq 1 \mathcal{L}^{n}$-a.e. in $\Omega, v_{k} \in W^{1, p}(\Omega)$, and $v_{k} \rightarrow 1$ in $L^{1}(\Omega)$.

Let us proceed with the computation. The sequence $F_{k}\left(u_{k}, v_{k}\right)$ can be written now as

$$
\begin{aligned}
F_{k}\left(u_{k}, v_{k}\right)= & \int_{\Omega} v_{k}\left|\nabla u_{k}\right|^{2} d x+\int_{\Omega \backslash\left(B_{k} \cup B_{k}^{\prime}\right)} \frac{\psi\left(v_{k}\right)}{\delta_{k}} d x+\int_{B_{k}}\left(\frac{\psi\left(v_{k}\right)}{\delta_{k}}+c \epsilon_{k}^{p-1}\left|\nabla v_{k}\right|^{p}\right) d x \\
& +\int_{B_{k}^{\prime}}\left(\frac{\psi\left(v_{k}\right)}{\delta_{k}}+c \epsilon_{k}^{p-1}\left|\nabla v_{k}\right|^{p}\right) d x .
\end{aligned}
$$

We have already computed the first and the second term of the previous expression in [10, Theorem $3.3]$ and in (4.18), showing that when $0<\alpha<\infty$ we find

$$
\int_{\Omega} v_{k}\left|\nabla u_{k}\right|^{2} d x+\int_{\Omega \backslash\left(B_{k} \cup B_{k}^{\prime}\right)} \frac{\psi\left(v_{k}\right)}{\delta_{k}} d x \leq \int_{\Omega}|\nabla u|^{2} d x+b_{\alpha} \int_{J_{u}}|[u]| d \mathcal{H}^{n-1}+o(1) ;
$$

whereas if $\alpha=0$ we find

$$
\int_{\Omega} v_{k}\left|\nabla u_{k}\right|^{2} d x+\int_{\Omega \backslash\left(B_{k} \cup B_{k}^{\prime}\right)} \frac{\psi\left(v_{k}\right)}{\delta_{k}} d x \leq \int_{\Omega}|\nabla u|^{2} d x+o(1) .
$$

Let us consider now the integral on $B_{k}$ in (5.2). By the choice of $B_{k}$ and $v_{k}$ we obtain

$$
\begin{aligned}
\int_{B_{k}}\left(\frac{\psi\left(v_{k}\right)}{\delta_{k}}+c \epsilon_{k}^{p-1}\left|\nabla v_{k}\right|^{p}\right) d x & =2(c p)^{\frac{1}{p}}\left(\frac{q \epsilon_{k}}{\delta_{k}}\right)^{\frac{1}{q}} \int_{J_{u}}\left[\int_{0}^{\mu_{k}} \psi\left(w_{k}\right)^{\frac{1}{q}} w_{k}^{\prime} d x_{n}\right] d \mathcal{H}^{n-1}(\bar{x}) \\
& =2(c p)^{\frac{1}{p}}\left(\frac{q \epsilon_{k}}{\delta_{k}}\right)^{\frac{1}{q}}\left(\int_{\eta_{k}}^{1-\rho_{k}} \psi^{\frac{1}{q}} d s\right) \mathcal{H}^{n-1}\left(J_{u}\right) .
\end{aligned}
$$

Moreover coarea formula implies

$$
\begin{aligned}
& \int_{B_{k}^{\prime}}\left(\frac{\psi\left(v_{k}\right)}{\delta_{k}}+c \epsilon_{k}^{p-1}\left|\nabla v_{k}\right|^{p}\right) d x \leq \\
\leq & c_{1}\left(\frac{\epsilon_{k}}{\delta_{k}}\right)^{\frac{1}{q}} \int_{\sigma_{k}^{\alpha}}^{\sigma_{k}^{\alpha}+\mu_{k}} \psi\left(w_{k}\left(t-\sigma_{k}^{\alpha}\right)\right)^{\frac{1}{q}} w_{k}^{\prime}\left(t-\sigma_{k}^{\alpha}\right) \mathcal{H}^{n-1}\left(\left\{d\left(x, \partial J_{u}\right)=t\right\}\right) d t \\
\leq & c_{2}\left(\sigma_{k}^{\alpha}+\mu_{k}\right)\left(\frac{\epsilon_{k}}{\delta_{k}}\right)^{\frac{1}{q}} \int_{0}^{\mu_{k}} \psi\left(w_{k}\right)^{\frac{1}{q}} w_{k}^{\prime} d t \\
\leq & c_{3}\left(\sigma_{k}^{\alpha}+\mu_{k}\right)\left(\frac{\epsilon_{k}}{\delta_{k}}\right)^{\frac{1}{q}}
\end{aligned}
$$

where $c_{1}, c_{2}, c_{3}<\infty$ are constant and we have used the fact that $\mathcal{H}^{n-1}\left(\left\{d\left(x, \partial J_{u}\right)=t\right\}\right)=O(t)$. The last term in (5.5) tends to 0 by the choice of $\beta, \sigma_{k}^{\alpha}$, and $\mu_{k}$. By (5.3), (5.4), (5.5), and (5.6) we obtain (3.14).

In the general case when $u \notin L^{\infty}(\Omega)$, we obtain (3.14) through a truncation argument. 
Let now $0<\alpha<\infty, \beta=\infty$; as in the case $n=1$ it is sufficient to prove by [6, Theorem 3.1] that for every $u \in S B V^{2}(\Omega)$ we have

$$
F_{\alpha, \infty}^{\prime \prime}(u, 1) \leq \int_{\Omega}|\nabla u|^{2} d x+b_{\alpha} \int_{J_{u}}|[u]| d \mathcal{H}^{n-1} .
$$

We define all parameters as in the previous case; the computations in (5.3), (5.4), and (5.6) give the same results as before, whereas the last term in (5.5) tends to 0 since $\beta=\infty$. Estimate (3.14) follows.

We conclude the proof of the estimate from above by studying the case $\alpha=0, \beta=\infty$. We shall prove that $F_{0, \infty}^{\prime \prime}(u, 1)=0$ for every $u \in L^{1}(\Omega)$.

Since $F_{0, \infty}^{\prime \prime}$ is lower semicontinuous, it is sufficient to prove the estimate on a set which is dense in $L^{1}(\Omega)$. To this aim we consider the set of functions which are constant on finitely many disjoint balls and null otherwise. For simplicity we consider only the case of a function $u$ which is constant on a ball $B$ well-contained in $\Omega$ and null out of $A$. Let $\sigma_{k}^{0}, \rho_{k}, w_{k}$, and $\mu_{k}$ be defined as before; let $\varphi_{k}$ be a cut-off function such that $\varphi_{k}=1$ on $(\partial B)_{\sigma_{k}^{0} / 2}, \varphi_{k}=0$ out of $(\partial B)_{\sigma_{k}^{0}}$, and $\left|\nabla \varphi_{k}\right| \leq 4 / \sigma_{k}^{0}$, where $(\partial B)_{r}:=\{d(x, \partial B)<r\}$. We define $u_{k}:=\left(1-\varphi_{k}\right) u$ and $v_{k}$ as $\eta_{k}$ on $(\partial B)_{\sigma_{k}^{0}}$, as $1-\rho_{k}$ out of $(\partial B)_{\sigma_{k}^{0}+\mu_{k}}$, and as $w_{k}\left(d(x, \partial B)-\sigma_{k}^{0}\right)$ in $(\partial B)_{\sigma_{k}^{0}+\mu_{k}} \backslash(\partial B)_{\sigma_{k}^{0}}$. By this choice $u_{k} \in H^{1}(\Omega), v_{k} \in V_{\eta_{k}}$ and $\left(u_{k}, v_{k}\right) \rightarrow(u, 1)$ in $L^{1}(\Omega) \times L^{1}(\Omega)$. Let us proceed with the computation.

We have that

$$
\begin{aligned}
\int_{\Omega} v_{k}\left|\nabla u_{k}\right|^{2} d x+\int_{(\partial B)_{\sigma_{k}^{0}}} \frac{\psi\left(v_{k}\right)}{\delta_{k}} d x & \leq\left(16 u^{2} \frac{\eta_{k}}{\left(\sigma_{k}^{0}\right)^{2}}+\frac{\psi\left(\eta_{k}\right)}{\delta_{k}}\right) \mathcal{L}^{n}\left((\partial B)_{\sigma_{k}^{0}}\right) \\
& \leq c_{1} \frac{\eta_{k}}{\sigma_{k}^{0}}+c_{2} \frac{\sigma_{k}^{0}}{\delta_{k}}
\end{aligned}
$$

where $c_{1}, c_{2}$ are constant; the last term in the previous expression tends to 0 by the choice of $\sigma_{k}^{0}$.

Since $\rho_{k}$ satisfies (4.13) we also obtain

$$
\int_{\Omega \backslash(\partial B)_{\sigma_{k}^{0}+\mu_{k}}} \frac{\psi\left(1-\rho_{k}\right)}{\delta_{k}} d x \leq o(1) .
$$

Finally we note that

$$
\begin{aligned}
\int_{(\partial B)_{\sigma_{k}^{0}+\mu_{k}} \backslash(\partial B)_{\sigma_{k}^{0}}}\left(\frac{\psi\left(v_{k}\right)}{\delta_{k}}+c \epsilon_{k}^{p-1}\left|\nabla v_{k}\right|^{p}\right) d x & =c_{3}\left(\frac{\epsilon_{k}}{\delta_{k}}\right)^{\frac{1}{q}} \int_{0}^{\mu_{k}} \psi\left(w_{k}\right)^{\frac{1}{q}} w_{k}^{\prime}\left(t+\sigma_{k}^{0}\right)^{n-1} d t \\
& \leq c_{4}\left(\frac{\epsilon_{k}}{\delta_{k}}\right)^{\frac{1}{q}},
\end{aligned}
$$

where $c_{3}, c_{4}$ are constant; since $\beta=\infty$ also the last term in the previous expression tends to 0 . Equality $F_{0, \infty}^{\prime \prime}(u, 1)=0$ follows.

\section{Convergence of Minimizers}

We conclude this paper by proving Theorem 1.2. Since this proof is quite similar to the one of [10, Theorem 7.1], we only sketch it focusing on the novelties. First we provide a compactness result.

Lemma 6.1. Let $\alpha>0$ or $\beta<\infty$. Let $\left(u_{k}, v_{k}\right)$ be a sequence in $L^{1}(\Omega) \times L^{1}(\Omega)$ such that $\left(u_{k}\right)$ is bounded in $L^{1}(\Omega)$ and

$$
\liminf _{k \rightarrow \infty} F_{k}\left(u_{k}, v_{k}\right)<\infty .
$$

Then there exists a subsequence $\left(u_{j}, v_{j}\right)$ of $\left(u_{k}, v_{k}\right)$ and a function $u \in G S B V(\Omega) \cap L^{1}(\Omega)$ such that $u_{j} \rightarrow u \mathcal{L}^{n}$-a.e. on $\Omega$ and $v_{j} \rightarrow 1$ in $L^{1}(\Omega)$.

If $0<\alpha<\infty$ and $\beta=\infty$, or $\alpha=\infty$, the convergence $u_{j} \rightarrow u$ is also in $L^{1}(\Omega)$.

The previous lemma does not apply when $\alpha=0$ and $\beta=\infty$, but we shall be able to prove Theorem 1.2 also in this case. 
Proof. We can suppose, up to subsequences, that $F_{k}\left(u_{k}, v_{k}\right)$ is bounded; in particular then $v_{k} \rightarrow 1$ in $L^{1}(\Omega)$. Now, when $0 \leq \alpha<\infty$ and $0 \leq \beta<\infty$ we can argue as in [10, Theorem 7.4].

Let $\alpha=\infty$. Repeating the computation in (4.1) we deduce by assumptions that $\left(u_{k}\right)$ is bounded in $B V(\Omega)$. This implies the existence of a function $u$ to which $u_{k}$ converges in $L^{1}(\Omega)$ and $\mathcal{L}^{n}$-a.e. in $\Omega$, up to subsequences. The same argument works in the case $0<\alpha<\infty, \beta=\infty$.

We also remark that we can define the sequence of functionals

$$
G_{k}(u, v):=F_{k}(u, v)+\int_{\Omega}|u-g|^{r} d x
$$

with $u, v \in L^{1}(\Omega)$, and we can easily prove, as in [10, Lemma 7.5], that $G_{k} \Gamma$-converges in $L^{1}(\Omega) \times L^{1}(\Omega)$ to the functional

$$
G_{\alpha, \beta}(u, v):=F_{\alpha, \beta}(u, v)+\int_{\Omega}|u-g|^{r} d x,
$$

where $u, v \in L^{1}(\Omega)$.

Let us prove now Theorem 1.2.

Proof. If $\alpha>0$ or $\beta<\infty$ we can argue as in [10, Theorem 7.1] through Lemma 6.1.

We suppose now that $\alpha=0$ and $\beta=\infty$. We fix $k$ and we consider a minimizer $\left(u_{k}, v_{k}\right) \in$ $H^{1}(\Omega) \times V_{\eta_{k}}$ of $G_{k}$. Since $G_{k}\left(u_{k}, v_{k}\right)$ is bounded, we can find a subsequence of $u_{k}$, not relabeld, and a function $u \in L^{r}(\Omega)$ to which $u_{k}$ converges weakly in $L^{r}(\Omega)$. Therefore we have

$$
\int_{\Omega}|u-g|^{r} d x \leq \liminf _{k \rightarrow \infty} \int_{\Omega}\left|u_{k}-g\right|^{r} d x \leq \liminf _{k \rightarrow \infty} G_{k}\left(\tilde{u}_{k}, \tilde{v}_{k}\right)=\int_{\Omega}|u-g|^{r} d x
$$

where we have chosen $\left(\tilde{u}_{k}, \tilde{v}_{k}\right) \rightarrow(u, 1)$ in $L^{1}(\Omega) \times L^{1}(\Omega)$ with $\lim _{k \rightarrow \infty} G_{k}\left(\tilde{u}_{k}, \tilde{v}_{k}\right)=G_{\alpha, \beta}(u, 1)$.

Since now $u_{k}-g \rightarrow u-g$ weakly in $L^{r}(\Omega)$ and $\left\|u_{k}-g\right\|_{L^{r}(\Omega)} \rightarrow\|\| u-g \|_{L^{r}(\Omega)}$ we also conclude that $u_{k} \rightarrow u$ strongly in $L^{r}(\Omega)$.

By the $\Gamma$-convergence of $G_{k}$ to $G_{\alpha}$ and by a general property of $\Gamma$-convergence (see [9, Corollary 7.20]), we find that $(u, 1)$ is a minimizer for $G_{\alpha}$ (so that $u=g \mathcal{L}^{n}$-a.e. in $\Omega$ ) and we get the convergence of minimum values and of minimizer in $L^{1}(\Omega) \times L^{1}(\Omega)$.

\section{ACKNOWLEDGMENTS}

The author wishes to thank Gianni Dal Maso for many useful conversations. This paper was partially supported by the Project "Variational Problems with Multiple Scales" 2008 of the Italian Ministry of Education, University, and Research.

\section{REFERENCES}

[1] L. Ambrosio, L. Fusco, and D. Pallara, "Functions of Bounded Variation and Free Discontinuity Problems", Oxford University Press, Oxford, 2000.

[2] L. Ambrosio, A. Lemenant, and G. Royer-Carfagni, A variational model for plastic slip and its regularization via Г-convergence, Preprint Scuola Normale, Pisa, 2010. Available from: http://cvgmt.sns.it/papers/amblemroy/.

[3] L. Ambrosio, V. M. Tortorelli, Approximation of functionals depending on jumps by elliptic functionals via Г-convergence, Comm. Pure Appl. Math. 43 (1990), 999-1036.

[4] L. Ambrosio, V. M. Tortorelli, On the approximation of free discontinuity problems, Boll. Un. Mat. Ital. 6-B (1992), 105-123.

[5] B. Bourdin, G.A. Francfort, and J.-J. Marigo, The variational approach to fracture, J. Elasticity 91 (2008), $5-148$.

[6] G. Bouchitt, A. Braides and G. Buttazzo, Relaxation results for some free discontinuity problems, J. reine angew. Math. 458 (1995), 1-18.

[7] G. Buttazzo, "Semicontinuity, Relaxation and Integral Representation in the Calculus of Variation", Pitman Res. Notes Math. Ser. 203, Longman, Harlow, 1989.

[8] G. Cortesani, R. Toader, A density result in SBV with respect to non-isotropic energies, Nonlinear Anal. 38 (1999), 585-604.

[9] G. Dal Maso, "An Introduction to Г-Convergence", Birkhäuser, Basel, 1993.

[10] G. Dal Maso, F. Iurlano, Fracture models as $\Gamma$-limits of damage models, to appear on Commun. Pure Appl. Anal. Available from http://cvgmt.sns.it/media/doc/paper/319/DM-Iur-11-CPAA.pdf. 
[11] R. Hill, "The Mathematical Theory of Plasticity", Clarendon Press, Oxford, 1950.

[12] K. Pham, J.-J. Marigo, C. Maurini, The issues of the uniqueness and the stability of the homogeneous response in uniaxial tests with gradient damage models, J. Mech. Phys. Solids 59 (2011), 1163-1190.

E-mail address: iurlano@sissa.it 